\title{
Architecture for Node-level Congestion in WSN using Rate Optimization
}

\author{
Mr. Bharath kumara ${ }^{1}$, Mr. Murthi Mahadeva Naik G ${ }^{2}$ \\ ${ }^{1}$ IV Semester, M.Tech, Malnad College of Engg, Hassan, India \\ ${ }^{2}$ Associate Professor, Dept. Of E\&C Engineering. Malnad College of Engg, Hassan, India
}

\begin{abstract}
The Application has presented the details of wireless sensor network differs basically from the overall knowledge network. It spotlight on tight communication however restricted in storage, lifetime, power and energy. The WSNs consists of unbelievable network load and it leads to energy wastage and packet loss. Several of the present concepts are developed for link level congestion management. The Rate optimization technique for node level congestion can assist to control the traffic at node level. Except supply and sink node the remaining nodes might participate in forwarding the packets towards the communication direction. The speed based mostly adjustment technique is applied to avoid packet dropping in order to save lots of the network resources. We have a tendency to be proposing this scheme to avoid the buffer overflow and it's not taking an excessive amount of energy consumption within the communication. This theme can assist to enhance the throughput, potency and resource saving. Node level congestion management is effectively required for WSN, because the node deployment will be anywhere. We are Introducing this theme using the network simulators extended tool referred to as mannerism.
\end{abstract}

Keywords- Buffer Overflow; Congestion Control; Node deployment; sink; source.

\section{Introduction}

Wireless sensor Networks are used tremendously in target monitoring, location tracking and battlefield etc. typically the sensor nodes are restricted in storage, lifetime, power, computation capability and in energy too. It can be deployed anywhere, where it would not be doable to use ancient networks. The formation of the network is done without human intervention. Equally addition and removal of nodes is finished while not human facilitate. We can operate these nodes in uninterrupted setting so as to accomplish the task. The sensor nodes are terribly little in nature, they consists of little processing unit, that has the capability of restricted computational power [1]. Generally they are battery powered and energy constrained. The sink node i.e. target node is most powerful that is employed as gateway to the wired network and its doing knowledge collecting and processing. The nodes deployed space is named sensing field, where every node has its own vary. The lifetime of the sensor node is generally restricted, before its energy exhausted, it ought to be used effectively. In several of the applications, less cost sensors are used and placed within the vast region [2]. The routing technique used is challengeable one. The topology conjointly random topology since it's changing its structure frequently. During the communication flow, traffic is unavoidable one. The traffic in WSNs causes the congestion within the network.

In previous analysis fairness bandwidth allocation isn't well addressed. Congestion in WSNs has negative impacts on network performance and application objective, i.e., packet loss, increased packet delay, wasted node energy and severe reliability degradation [3].

However, some characteristics of WSNs, such as constrained resources, interference of methods and also the lack of centralized coordination, create the congestion down side in WSNs over ancient networks. Additionally, to let the sink successfully receive the information from totally different sensors, we need to contemplate the fairness issue among the supply nodes [4]. Increasing network competition causes an increase in packet collisions within the wireless medium. After Several unsuccessful retransmissions, packets are dropped at the sender node. So as to regulate the congestion initially congestion detection is crucial.

In WSNs, Congestion is detected by 2 main factors like buffer overflow and link collisions. To realize the congestion less communication in sensor networks, we've to try to rate based mostly adjustment within the communication path. have a tendency to be introducing all the intermediate nodes beneath. The nodes which are surrounding the supply and sink are mainly categorized. The nodes that are surrounding the supply node are referred to as supply surrounding nodes and therefore the nodes that all are terribly with regards to sink is termed sink surrounding nodes. These intermediate nodes are getting to management the truthful information rate within the communication. The wireless sensor network not nevertheless reached high, since it's having drawback of flow management, rate management, congestion management, medium access management, queue management, power management and topology management. It is very difficult to supply complete resolution for solving of these problems. The remaining a part of this paper is continued with connected works, models and issues, proposed scheme and analysis. Finally we have a tendency to conclude this paper. 


\section{NETWORK FRAMEWORK}

Wireless sensor nodes are deployed randomly in sensing field; the nodes share the wireless medium to speak with neighbor nodes. Usually sink is also placed anywhere within the network. So as to contact the sink it needs to communicate through the intermediate nodes. The intermediate nodes become busy, once they are attempting to forward the packets quite its capability. If it receives additional information than it will forward then congestion occurs within the network. Therefore the characteristics depend upon the generating rate of the data's from the supply node. If the generating rate is low then the interference makes less severe. The overall architecture of the proposed system is shown in Figure 1.

The nodes face the difficulties to search out the optimal path towards the sink node. It passes the packet through the intermediate nodes. The mediating nodes are wasting their energy so as to search out the sink node, if the target is simply too way from the source nodes.

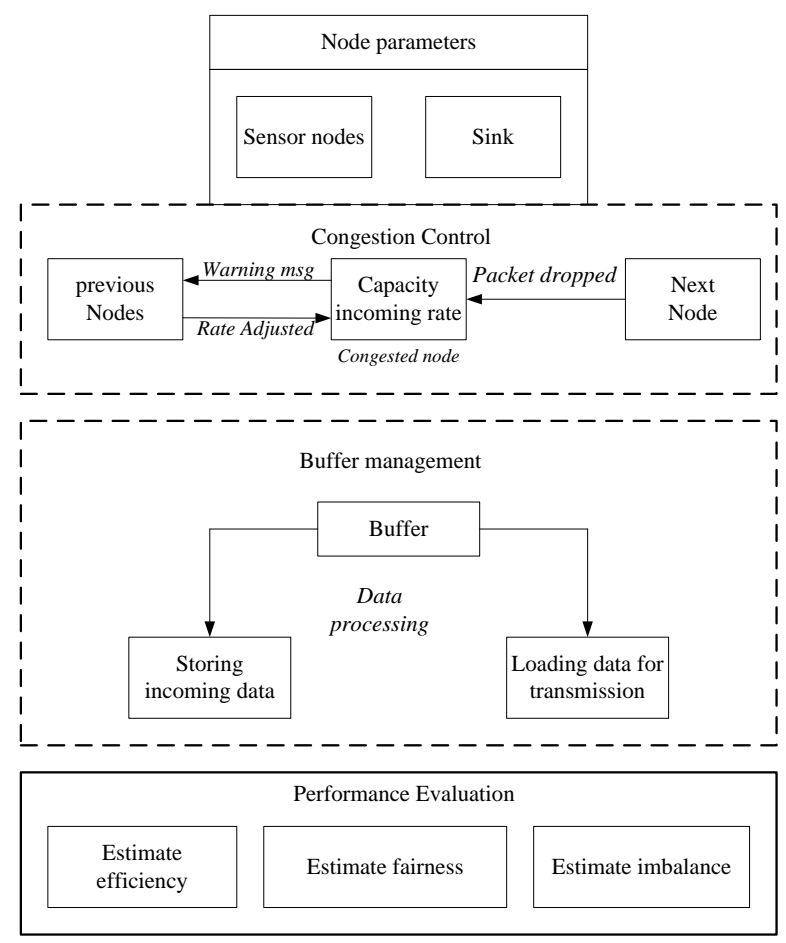

Figure 1: Overall Architecture of proposed system.

Normally the buffer is used for storing the data temporarily, so the node's buffer size is main consideration within the network. If size of the buffer is full the packet will dropped, then concluded as congestion in the node. once congestion occurred warning message is sent to source nodes about there packet generation. Else the packet will received from the source nodes.

The congestion management is followed to induce rid of the congestion. However the congestion avoidance is employed for preventing congestion. When the network load will increase the packet loss attainable conjointly increased. To regulate the sending rate of the info packets from the supply and intermediate nodes, we've got to regulate the generating rate of packets. The efficient rate allocation could assist to regulate the congestion in the network. In networking concept, network congestion happens when a link or node is carrying most information in order that its quality of service is degraded. Typical effects embody queuing delay, packet loss or the blocking of latest connections.

There are two reasons mainly affecting the network throughput that results in congestion within the communication. Those factors are buffer overflow and link collision. In order to control the congestion typically two techniques are followed within the network ideas. Resource management and traffic management are the techniques handled to manage the congestion.

\section{Proposed System}

For management the node level congestion, the node is identified initially that has met the congestion. Controlling logic is applied to that exact node to urge eradicated from the traffic. The supply surrounding nodes initially receives the packets from the supply nodes. The source nodes could choose any of the supply surrounding nodes to forward the packet towards the sink node. 
The congested node drops a number of the packets and it receives the warning message from subsequent nodes. Now the congested node realizes that it's dropped a number of the packets. The message results in consequence that provides warning message to the supply node.

The warning message is shipped when the packet is dropped or the nodes receive quite they will forward. So as to adjust or update the information rate the speed optimization is applied. Our aim is to regulate the buffer overflow. When the incoming rate is bigger than its capability then it ends up in overflow. Now the supply node adjusts its information generating rate so as to avoid the buffer overflow within the communication. The algorithm used for this theme is described below. Each sensor node watches its buffer and notices the congestion bit. The buffer size is measured in terms of packets and information rate is measured by variety of packets transmitted per unit time. The algorithm illustrates that, each sensor node has individual buffer, and its buffer level is continuously listened. The minimum and maximum threshold levels of the buffer occupancy known as MinBo, MaxBo respectively. The present buffer occupancy is indicated by CBo and it's compared with extreme values. If the present level is exceeding the most extreme means that then set the congestion notification bit within the packet header. So the previous node can decrease the transmitting rate. Similarly if gift worth goes beyond the minimum extreme then the previous node will update its transmitting rate. The process flow of the proposed system is as shown in Figure 2.

The buffer has two parts normally, one is for storing the incoming knowledge and another is for loading the info for transmission. Each node is processing the incoming knowledge and the processed knowledge is place into the loading portion. If the incoming rate is slow, the info is well processed. If the incoming rate is just too quick, the node fails to method the info properly and dropping a number of the packets.

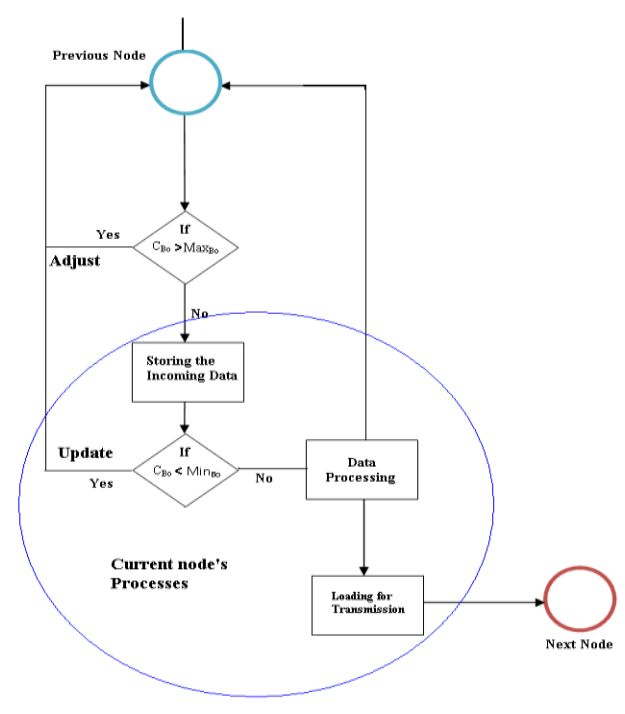

Fig 2: process flow of the work.

\section{Results and Analysis}

The proposed system is implemented on 32 bit windows OS with $1.84 \mathrm{GHz}$ processor. The simulation is created on Matlab. We have a tendency to implement the simulation surroundings for comparison purpose. The network performance will be determined by few terms such as channel busy time, channel utilization level, efficiency, fairness and imbalance. The quantity of the time the channel is allotted for packet transmission and reception is called channel busy time. Equally channel is typically being idle throughout communication. The channel or medium utilization level will be outlined as average rate of reliable packets delivered through the channel. The result for communication considering congestion is shown in Figure 3. 


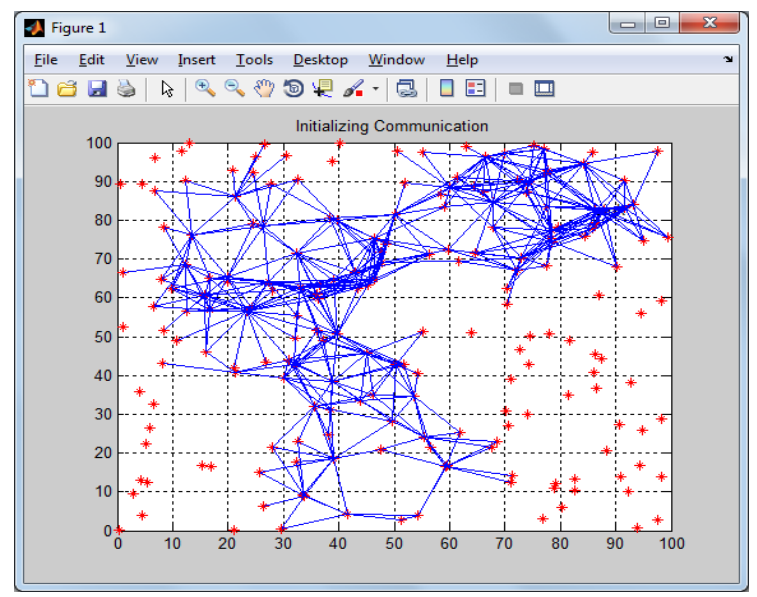

Figure 3: Initializing WSN with congestion

The MAC layer utilization level will be determined by noticing whether or not the medium is busy or idle. The binary values are used for indicating MAC layer utilization level. 1, 0 are used for indicating channel is currently busy or idle respectively. The most issue deciding buffer overflow is interface queue length. The additional quantity of packet are buffered within the interface queue solely, the packets are dropped when the queue length is restricted within the network. The main terms that are to be calculated to see the network performance are potency, fairness and imbalance. The efficiency of the communication is calculated by variety hops the successful packets travelled to the entire variety of packets placed (dropped and retransmitted conjointly included) within the network. However, mesh architecture is design to depict the congestion as shown in Figure 4. The simulation study shows that congestion to be highly minimized with adjustment of data rate.

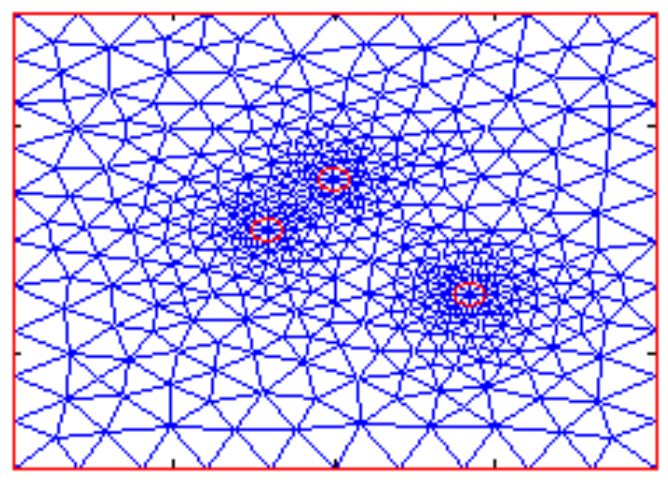

Figure 4: Identification of congested area in WSN

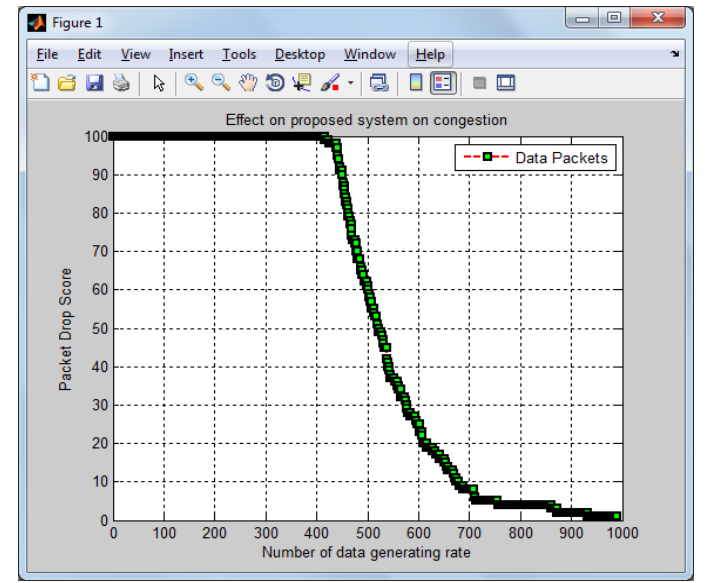

Figure 5: Effect of proposed system on packet drop. 
The simulation results in figure 5. shows the dropping ratio of the 'Without congestion management' and congestion control Scheme. The figure shows the packet dropping price in 3 dimensional views. Our theme offers affordable improvement in throughput and reduced packet loss. In congestion management theme the buffer overflow is principally avoided so as to scale back the packet loss. Proposed theme allocates the truthful rate to avoid the congestion and improves the communication.

\section{CONCLUSIONS}

In this Conclusion we have planned to manage the node level congestion within the wireless sensor networks. This theme achieves affordable honest knowledge rate within the communication. The simulation theme shows higher performance in terms of efficiency, reliability and resource saving. Notably for improving throughput it'll assist whereas network load is increased. This theme becomes reliable for avoiding packet loss throughout the congested node placed within the communication. In order to create the honest rate among the nodes, this theme is applicable one. It aids to manage congestion at node level particularly at intermediate nodes.

\section{REFERENCES}

[1] "A Survey on Congestion Control in Wireless Sensor Networks" Rekha Chakravarthi1, C. Gomathy2, Suraj K. Sebastian3, Pushparaj. K4, Vinu Binto Mon5- International Journal of Computer Science \& CommunicationVol. 1, No. 1, Jan-Jun 2010.

[2] Wang, Q. (2010). "Traffic Analysis, Modeling and Their Applications in Energy-Constrained Wireless Sensor Networks-On Network Optimization and Anomaly Detection." Mid Sweden University, Sweden ISBN 978-91- 86073-64-0, ISSN 1652-893x.

[3] J. Kulik, W. Rabiner, and H. Balakrishnan, "Adaptive protocols for information dissemination in wireless sensor networks," in Proc. ACM MobiCom, Seattle, WA, Aug. 1999, pp. 174-185.

[4] S. Chen, Y. Fang, and Y. Xia, "Lexicographic maxmin fairness for data collection in wireless sensor networks," IEEE Trans. Mobile Comput., vol. 6, no. 7, pp. 762-776, Jul. 2007.

[5] Chonggang Wang, Kazem Sohraby, Victor Lawrence, Bo Li, Yueming Hu "Priority-based Congestion Control in Wireless Sensor Networks" Proceedings of the IEEE International Conference on Sensor Networks, Ubiquitous, and Trustworthy Computing (SUTC'06) 0-7695-2553-9/06 \$20.00 @ 2006

[6] R.Then Malar "Congestion Control in Wireless Sensor Networks Based Multi-Path Routing In Priority Rate Adjustment Technique" Published in International Journal of Advanced Engineering \& Applications, Jan. 2010

[7] X. Liu: "Promoting Node-to-Node Congestion Control in Internet" Wireless and Mobile Computing, Networking and Communications (WiMob), 2010 IEEE 6th International Conference on

[8] Yong-min Liu, Xiao-hong NIAN and Wu-yi LU "Some Control Strategy relate to Congestion Control for Wireless Sensor Networks" 2009 International Conference on Computer Engineering and Applications IPCSIT vol.2 (2011) (C) (2011) IACSIT Press, Singapore

[9] Falko Dressler "Locality Driven Congestion Control in Self-Organizing Wireless Sensor Networks" rd International Conference on Pervasive Computing (Pervasive 2005):

[10] Haitao Zhao, Yuning Dong, Hui Zhang, and Xinxing Zhao "A State-Aware Rate Optimization Scheme based on Congestion Discrimination over Wireless Networks" JOURNAL OF COMPUTERS, VOL. 7, NO. 3, MARCH 2012, Manuscript received Sept. 25, 2010; revised Nov. 16, 2010; accepted July 31, 2011.

[11] Yufang Xi and Edmund M. Yeh "Node-Based Optimal Power Control, Routing, and Congestion Control in Wireless Networks" supported in part by Army Research Office (ARO) Young Investigator Program (YIP) grant DAAD19-03-1-0229 and by National Science Foundation (NSF) grant CCR-0313183

[12] C. Wang, B. Li,, K. Sohraby, M. Daneshmand "Upstream Congestion Control in Wireless Sensor Networks Through Cross-Layer Optimization" IEEE JOURNAL ON SELECTED AREAS IN COMMUNICATIONS, VOL. 25, NO. 4, MAY 2007.

[13] S.Surekha, C.Rajendra "Applications of Wireless Sensor Network by Avoiding Congestion" International Journal of Recent Technology and Engineering (IJRTE) ISSN: 2277-3878, Volume-1, Issue-2, June 2012

[14] Marcos Augusto M. Vieira, Luiz Filipe M. Vieira, Linnyer B. Ruiz, Antonio A.F. Loureiro, Antonio O. Fernandes, Jos’e Marcos S. Nogueira "Scheduling Nodes in Wireless Sensor Networks: A Voronoi Approach" 